\title{
sciendo
}

Transport and Telecommunication, 2021, volume 22, no. 3, 278-286

Transport and Telecommunication Institute, Lomonosova 1, Riga, LV-1019, Latvia

DOI 10.2478/ttj-2021-0021

\section{TRAJECTORY PLANNING OF AUTONOMOUS VEHICLE IN FREEWAY DRIVING}

\author{
Hashem Ghariblu ${ }^{1}$, Hossein B. Moghaddam ${ }^{2}$ \\ ${ }^{1,2}$ Mechanical Engineering Department, University of Zanjan, Zanjan, Iran \\ *Corresponding Author: ' ghariblu@znu.ac.ir, \\ ${ }^{2}$ hosseinb.moghaddam@znu.ac.ir
}

\begin{abstract}
This paper describes trajectory planning for an Autonomous Vehicle (AV) in the freeway path. Three types of driving modes are analyzed. First was free flow, this constitutes that moving at the desired speed is determined at the beginning of the movement. Second case was car following, when overtaking or lane-change was impossible, distance or speed adaptation is executed using the variable acceleration/deceleration strategy. Third case was lane change or overtaking. For lane change or overtaking paths, the 5th degree polynomial is used to create a curvilinear path to changes its path to the left lane and then returns to its default lane. The velocity and relative distances of cars are main factors for decision making. All proper driving decisions algorithm is introduced. According to autonomous car desired velocity, in the two driving cases (fast and slow desired velocity for AV) are studied by simulation and their results analyzed and compared with together.
\end{abstract}

Keywords: Trajectory Planning, Autonomous Vehicle, Freeway, Overtaking

\section{Introduction}

One major important area in the development of autonomous vehicles (AV) is solving their trajectory planning problems. This is in regards to enabling them to make proper decisions when they are facing with different situations, during their movement from start point to a destination. AV's have many advantages like increase in safety and comfort, reduction of human errors and accidents, reducing travelling time, and improving fuel economy and emissions.

Most important subsystems of autonomous vehicles are perception and mapping, trajectory planning, and vehicle control modules. The perception and mapping recognize the peripheral environment and detects obstacles and other vehicles by using sensors like cameras (Tarel et al., 2012, Zaarane et al., 2020), radars (Patole et al., 2017, Bilik et al., 2017) or Lidar (Maksymova et al., 2018, Dominiguez et al., 2011). Mapping process is done by using GPS and IMU (Milanes et al., 2008, Sukkarieh et al., 1999). Trajectory planning is generation of a path that conducts vehicle from an origin to destination while avoiding obstacles and other adjacent vehicles. Moreover, generated trajectory must satisfy the vehicle kinematics and dynamics constrains and passenger's comfort and safety. After environment perception and trajectory planning, controllers of an AV commands to the actuators like throttle/brake controller and steering control to control the longitudinal and lateral parameters of AV (Britting, 1999). Systems including Cruise control (CC) or adaptive cruise control (ACC) or cooperative adaptive cruise control (CACC) are used for longitudinal control (Kesting et al., 2007). The longitudinal control creates a suitable decision according to the behind and leader vehicle (LV). Alotibi et al. studied the Cooperative Adaptive Cruise Control (CACC) in autonomous vehicles where, vehicles regulate their speed according to a preceding leader vehicle in the lane, forming a platoon (Alotibi et al., 2020). Systems including lane keep assist (LKA) (Mameri et al., 2015), electronic stability control (ESC) are used for lateral control of AV and respectively create smooth lane change or overtaking (Chen et al., 2014). To model the lane changing and overtaking, kinematic like polynomial (Dixit et al., 2018, Zhang et al., 2013, Houenou et al., 2013) or sinusoidal (Kanaris et al., 2001) models describe the lane change maneuver in the form of equations. But polynomial trajectory curve is the most widely adopted for overtaking planning. Shim et al. have employed the six-order polynomials for overtaking trajectory planning (Shim et al., 2012). In general, polynomial functions with higher order for the overtaking trajectory create smoother variations for the state variables (e.g., position, velocity, and acceleration). But higher order polynomial function needs more input information to determine the model parameters. The freeway has generally three lanes and more, where the AV, depend on the speed determined by the passenger or by default (desired speed), would select to stay on a specific lane. When the AV encounters slower or faster vehicles it will decide to change lane and overtake, or to keep moving in current lane. The AV should move with safe distance and 
speed behind the LV. At any time of travelling, relative distance and speed must be measured and analysed. Mampearachchi et al., (2018) proposed a safe overtaking manoeuvre on two-lane highways, with a number of models. None of the preceding researches in the literature covered the movement of an autonomous vehicle in a longer period of time, which included various types of maneuvers, including cruise control; lane change and overtaking.

There are two common methods for trajectory planning, kinematic method and stochastic method. Kinematic method uses a polynomial equation to define a curved path. Shojaei introduced a trajectory planning with 5th degree polynomial and applied boundary conditions to define coefficient of 5th degree equation [6], Ghariblu introduced a trajectory planning with 4th degree polynomials. Skačkauskas et al. (2019) proposed a hybrid global path planning algorithm combined from both, classical and heuristic genetic algorithm approaches. To calculate value of the 4th coefficients, boundary conditions must be satisfied. The lateral velocity in the middle of the lane change is assumed to be maximum. At this moment the longitudinal velocity will be the average of velocity during the overtaking event.

Unlike earlier researches that only have analysed one of driving modes like overtaking or car following in a short time, main focus of this research is trajectory planning of an AV in freeway with three lanes and simulation of driver's behaviors in long time travelling. Here, it is assumed that all environment information like position of vehicles, obstacles, freeway boundaries and lane marking, are detected and measured at each state. Also, lane change is modelled by using a 5 th degree polynomial. The reminder of the paper is organized as follows. Section 2 defines the problem and describes vehicle behavior to different road conditions and adjacent vehicles. The trajectory planning algorithm for the AVs motion is presented in Section 3. In Section 4, the accuracy of the proposed algorithm is verified by two different simulations, and conclusions and results are given in Section 5.

\section{Problem Definition and Modelling}

\subsection{Problem definition}

The main focus of this research is trajectory planning of an AV in freeway and simulation of driver's behavior in the long time travelling. It is assumed that freeway is straight and there is no curvature in the path. Also, there is no merging, exit and crossing in the freeway. During the travelling, AV may encounter some slower or faster vehicles ahead. In some situations, AV may overtake the LV. If there are no overtaking conditions, AV adapts itself with the LV. In the various situations, AV must select an appropriate driving mode named as as free flow, car following (distance adaptation, speed adaptation), or overtaking. For each driving mode a set of conditions must be satisfied. The AV, should keep a safety distance $\left(h_{m}\right)$ from a LV [8]. Assuming similar deceleration rates for both LV and AV, the $h_{m}$ depends on maximum allowable speed of freeway's lane, length of AV and time constant. Minimum and maximum speed limits denoted by $V_{\min }$ and $V_{\max } \mathrm{AV}$ and other adjacent vehicles do not exceed freeway speed limits. Meanwhile, the desired velocity of AV and other adjacent vehicles are randomly selected in the range of freeway speed limits.

\subsection{Driving modes}

In this section, three types of driving modes and formulation will be presented.

Free Flow

According to freeway speed limit range, and environment conditions, passenger or AV itself selects a proper speed (desired speed) to keep moving in current lane. desired speed denotes by $V_{D}$. In this driving mode, AV's relative distance to LV is higher than safety distance $\left(h_{m}\right)$ and its speed is equal or lower than LV and there is no interaction between AV and other vehicles.

$V_{A V}=V_{D}$.

\section{Car following}

Car following divided in two modes, speed adaptation or distance adaptation.

\section{a) Speed adaptation}

If there is no safe gap between two vehicles in the left lane for overtaking and relative distance between AV and LV higher than safety distance $h_{m}$, and AV's velocity is higher than LV's velocity, speed adaptation must be done. At each time steps, deceleration depends on relative distance and velocity difference between $\mathrm{AV}$ and $\mathrm{LV}$, so deceleration is not linear and varies in each time step. Here 
unsymmetric Gazis-Herman-Rothery deceleration model [9], defined by Eqn. (2), is employed for acceleration/deceleration rate. This is obvious that deceleration is realized by braking and/or reducing the throttle opening.

$a_{A V}=\alpha \frac{V_{A V}^{\beta}}{\left(X_{L}-X_{A V}\right)^{l}}\left(V_{L}-V_{A V}\right)$,

where, $\alpha, \beta, l$ are positive model constant parameters and $X_{L}$ and $V_{L}$ is leader vehicle's position and velocity.

\section{b) Distance adaptation}

If relative distance between AV and LV is lower than $h_{m}$, and AV's velocity is higher than LV's velocity, distance adaptation must be done, by using brakes or reducing throttle opening, AV's velocity is decreased and relative distance between $\mathrm{AV}$ and $\mathrm{LV}$ is reached to $h_{m}$. Deceleration is also proportional with relative distance and velocity among AV and LV (Eqn. (2)).

\section{Overtaking}

If AV's velocity is higher than LV's velocity and gap $(D)$ between two vehicles in the left lane is sufficient and safe $\left(D_{\text {safe }}\right)$, overtaking will be done. Overtaking divided to three steps, first, lane change to new lane, second, keep moving in new lane until relative distance between AV and overtaken vehicle ( $\mathrm{LV}$ in previous lane) is equal or more, third, return to previous lane (Fig 1).

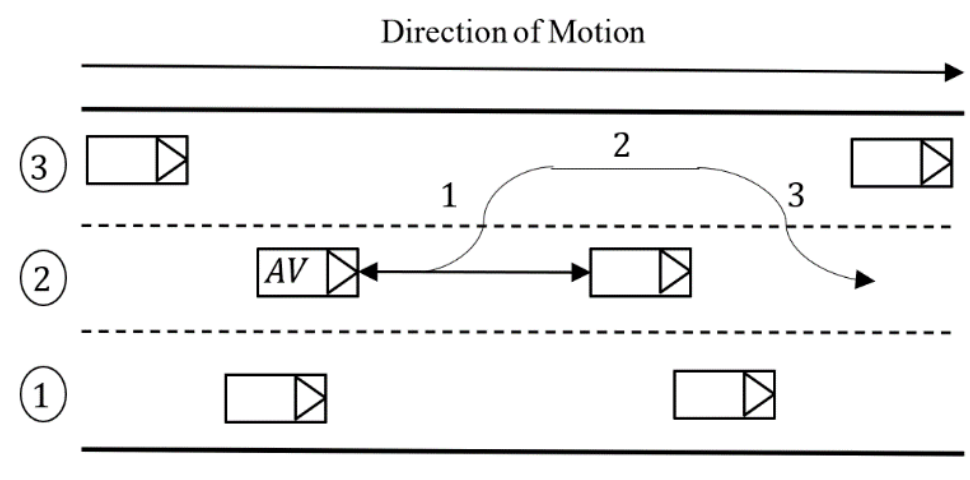

Figure 1. Three steps of AV's overtaking

In overtaking process, first and third step paths are curvilinear. Here, these paths are defined by a 5th degree time dependant polynomial (Eqn. (3)) [10].

$y(t)=d\left\{10\left(\frac{V_{A V} \times t}{x_{e}}\right)^{3}-15\left(\frac{V_{A V} \times t}{x_{e}}\right)^{4}+6\left(\frac{V_{A V} \times t}{x_{e}}\right)^{5}\right\}$

where, $\mathrm{d}$ is the overall lateral position between two adjacent lanes, $\mathrm{t}$ is the time, $\mathrm{y}$ is the lateral position at each time step, $x_{e}$ is longitudinal displacement during a complete lane change the end point of overtaking path. The $x_{e}$ in the Eqn. (3) is defined by Eqn. (4) as,

$x_{e}=V_{A V} \sqrt{\frac{d}{a_{y \max }}\left\{60 \frac{x_{m}}{x_{e}}-180\left(\frac{x_{m}}{x_{e}}\right)^{2}+120\left(\frac{x_{m}}{x_{e}}\right)^{3}\right\}}$,

where, $a_{y \max }$ is the maximum lateral acceleration that is considered equal to $0.05 \mathrm{~g}(\mathrm{~g}=9.81 \mathrm{~m} / \mathrm{s})$ in the simulation tests, $x_{m}$ is the longitudinal position where the curvature is maximum. Ratio of $x_{m}$ to $x_{e}$ calculated by (chee et al., 1994) that is equal to 0.2113 . Total time of lane changing is defined by (5).

$\mathrm{T}_{T}=\frac{x_{e}}{\mathrm{~V}}$.

\section{Trajectory Planning Algorithm}

In this section all decisions and driving modes have defined by an algorithm and simulation will be based on this algorithm. At the beginning of program at $t=0$, primary input parameters are, freeway 
speed limits $V_{\max }$ and $V_{\min }$, AV's desired velocity $\left(V_{D}\right)$, and adjacent vehicles relative distances and velocities (front, back left, front left of AV).

The following pseudo code shows driving modes in the freeway, and program runs until reaching to final executing time $T_{f}$.

\subsection{Algorithm of decision making}

It is Assumed that, AV's velocity and default lane, velocity and positions of adjacent vehicles are known and all vehicle speeds obey freeway speed limits. At every time step, program checks the condition and takes the best decision. In this section driving modes are divided into different cases and every case will be described in the following and are shown in a flowchart (Figure 2).

Case 1. If $X_{f} \geq h_{m}$

If $V_{A V} \leq V_{f}$, acceleration or keep moving with current speed until $V_{A V}=V_{D}$ and $X_{f}=h_{m}$.

If $V_{A V}=V_{D}$, free flow driving, there is no action, keep moving with desired velocity $\left(V_{D}\right)$.

Case 2. If $X_{f}<h_{m}$ and $V_{A V}<V_{f}$, no need action, because of the lower AV's velocity, relative distance increases until $X_{f}=h_{m}$ spontaneously.

Case 3. If $X_{f}<h_{m}$ and $V_{A V} \geq V_{f}$, checking the gap (D) between two vehicles in left lane.

3.1. If $D>D_{\text {safe }}$, by using Eqns (3) and (4) overtaking starts.

3.2. If $D \leq D_{\text {safe }}$, by using Eqn (2), distance adaptation starts.

Case 4. If $X_{f} \geq h_{m}$ and $V_{A V}>V_{f}$, checking the gap (D) between two vehicles in left lane.

4.1. If $D>D_{\text {safe }}$, by using Eqns (3) and (4) overtaking starts.

4.2. If $D \leq D_{\text {safe }}$, by using Eqn (2), speed adaptation starts.

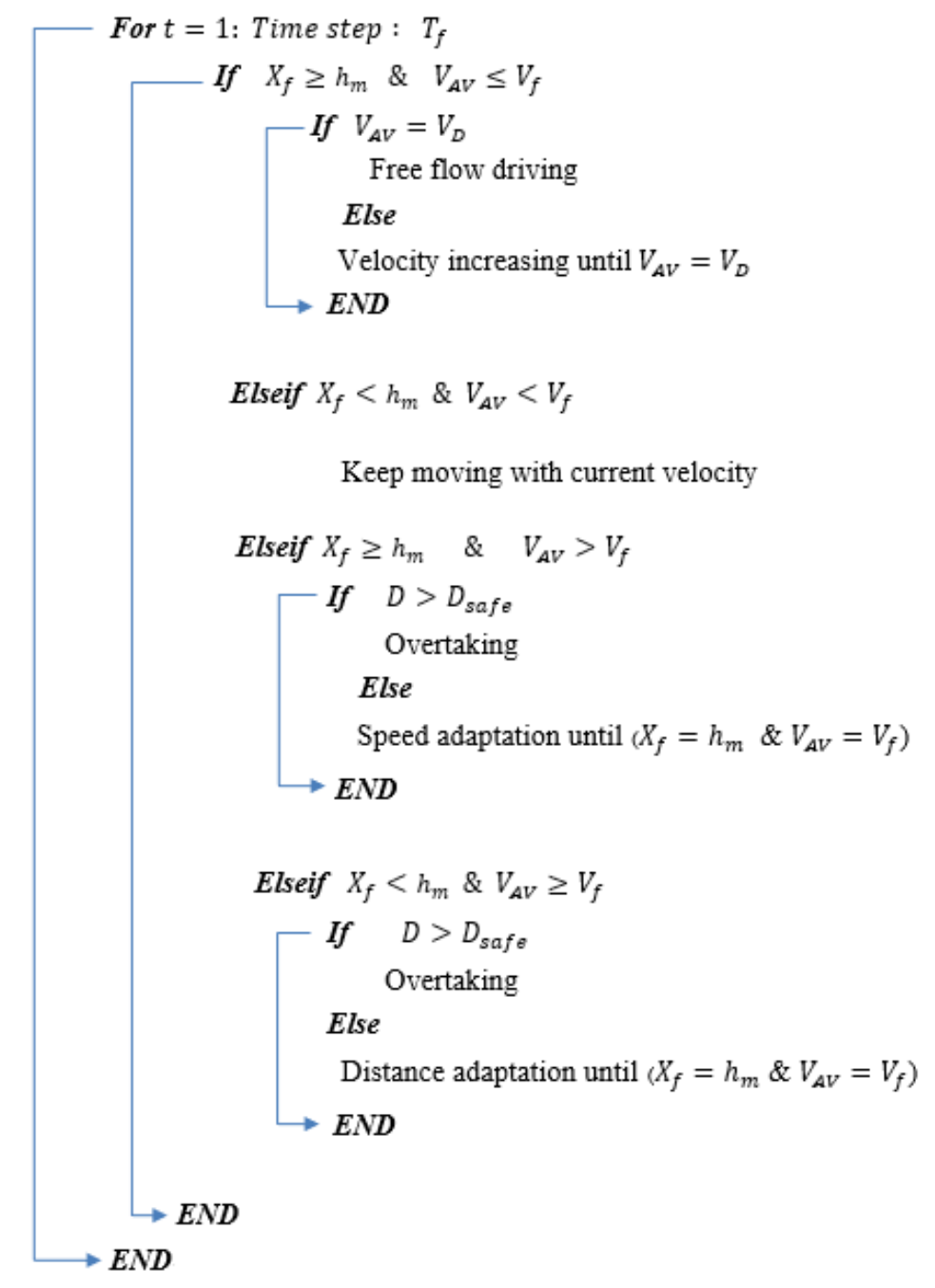




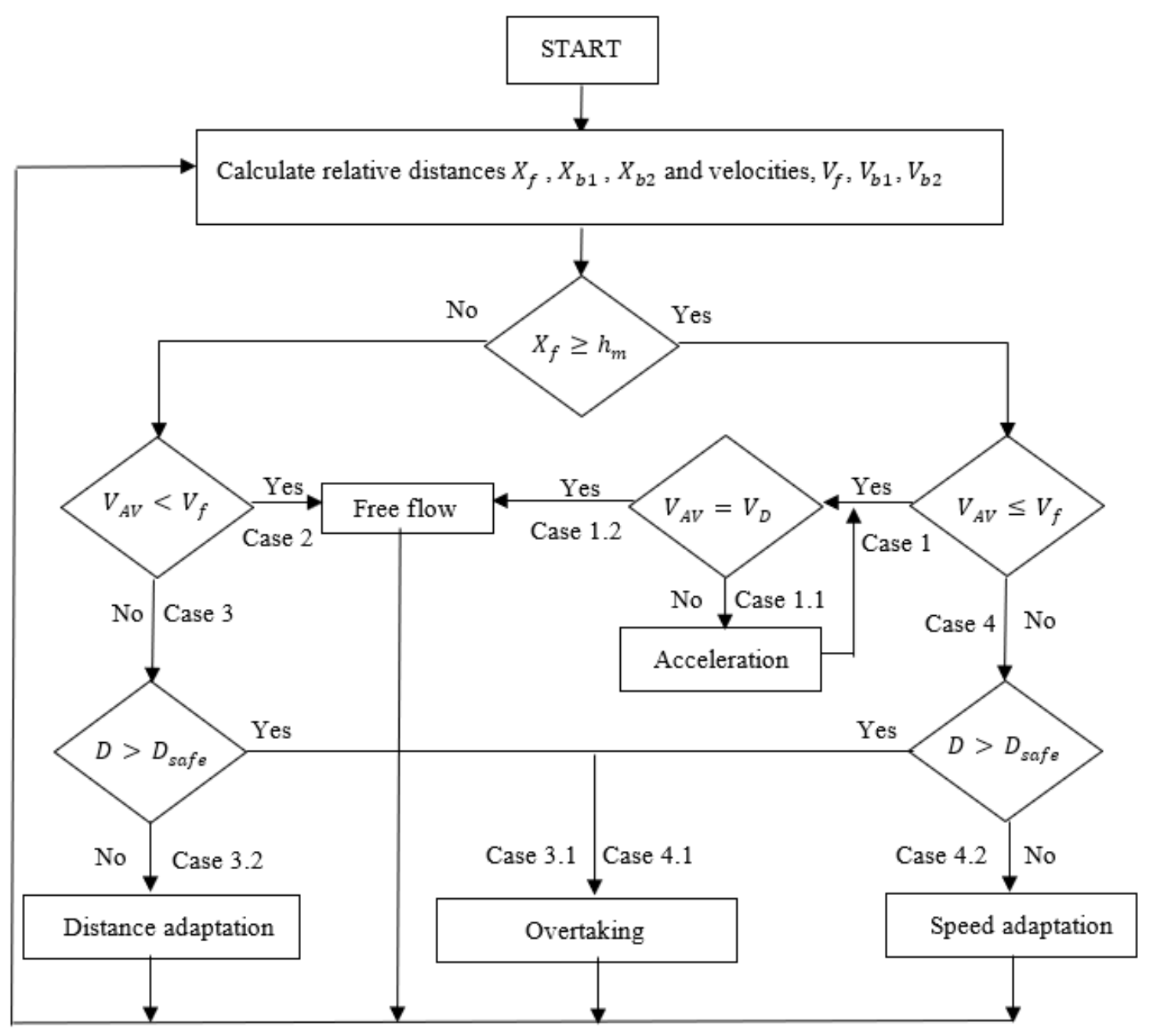

Figure 2. Decision making of driving modes

\section{Simulation Study}

Performing autonomous vehicles experiments in the real environment are risky and expensive, so a computer programming can simulate and investigate the autonomous vehicle moving strategies. Here, two different simulations executed with total simulation time $600 \mathrm{~s}(10 \mathrm{~min})$ and each lane width $4 \mathrm{~m}$. In the first simulation according to lane speed limit, AV starts at $X_{A V}=0 \mathrm{~m}$ and $y=2 \mathrm{~m}$ (lane 1 is default lane and lane 2 is overtaking lane). The allowable speed range in lane 1 is assumed between 80 and 100 $\mathrm{Km} / \mathrm{h}$. In the second simulation according to the lane speed limit, AV starts at $X_{A V}=0 \mathrm{~m}$ and $y=6 \mathrm{~m}$ (lane 2 is default lane and lane 3 is overtaking lane). At the beginning of simulation, adjacent vehicles are generated in distance between $0.5 X_{f}$ to $1.5 X_{f}$ randomly. When distance of adjacent vehicle reaches to $4 X_{f}$, program eliminate this vehicle and regenerates another vehicle in distance between $0.5 h_{m}$ to $1.5 h_{m}$ randomly.

\subsection{First simulation}

As depicted in Figure 3, when program starts to run, according to speed limits of lane 1, program selects by randomly AVs desired velocity $(96.1 \mathrm{Km} / \mathrm{h})$. AV starts moving with desired velocity in lane 1 . Safe distance $h_{m}$ between two vehicles is calculated and it's equal to $23.14 \mathrm{~m}$. At this time ( $t=0 \mathrm{~s}$ ), two vehicles in left lane with a defined velocity and distance from AV is generated, and a LV with initial velocity $80.6 \mathrm{Km} / \mathrm{h}$ and initial distance $35 \mathrm{~m}$ in front of $\mathrm{AV}$ is generated. AV's velocity is higher than LV's velocity and $X_{f}$ is higher than $h_{m}$, so AV checks distance between two vehicles $(D)$ in left lane for overtaking, and there is no safe distance $\left(D_{\text {safe }}\right)$ between two vehicles in the left lane, so AV must decelerate until $X_{f}=h_{m}$ and overtaking conditions are provided. In continue at $t=159 \mathrm{~s}$, (section A), 
overtaking conditions are provided and AV changes its lane to overtake. In second step of lane changing, as the AV is moving in lane 2, AV increase its velocity to reach $95.5 \mathrm{Km} / \mathrm{h}$.

At $t=187 \mathrm{~s}$ (section $\mathrm{B}$ ), lane changing has been done and AV has returned to previous lane (lane 1). At this time a new LV with velocity $93.4 \mathrm{Km} / \mathrm{h}$ and distance $25.56 \mathrm{~m}$ in front of $\mathrm{AV}$ is generated. Overtaking is impossible, so AV must decelerate until $X_{f}=h_{m}$ (speed adaptation), and AV's velocity reaches to $93.4 \mathrm{Km} / \mathrm{h}$ during deceleration and $X_{f}$ reaches to $24.40 \mathrm{~m}$, and AV keep moving with this velocity and holds its distance $X_{f}$ until $t=470 \mathrm{~s}$. At this time (section C), overtaking conditions provide and AV overtakes. when AV returns to previous lane (lane 1), at $t=501.5 \mathrm{~s}$, a new LV with velocity $96.6 \mathrm{Km} / \mathrm{h}$ and distance $14.93 \mathrm{~m}$ in front of AV is generated. AV's velocity is higher than LV and $X_{f}$ is lower than safe distance $h_{m}\left(X_{f}=14.93 \mathrm{~m}\right)$, and overtaking is impossible, so AV decelerates (distance adaptation) until its velocity reaches to $95.3 \mathrm{Km} / \mathrm{h}$ and $\mathrm{AV}$ keep moving with same velocity until its distance increases $\left(X_{f}=23.79 \mathrm{~m}\right)$ at $t=532.5 \mathrm{~s}$ (section D). After this section, LV's velocity is higher than AV's desired velocity, so AV can increase its velocity until desired velocity $(96.1 \mathrm{Km} / \mathrm{h})$ and keep moving until program stops at $t=600 \mathrm{~s}$. Other result of this simulation is presented in Table 1.

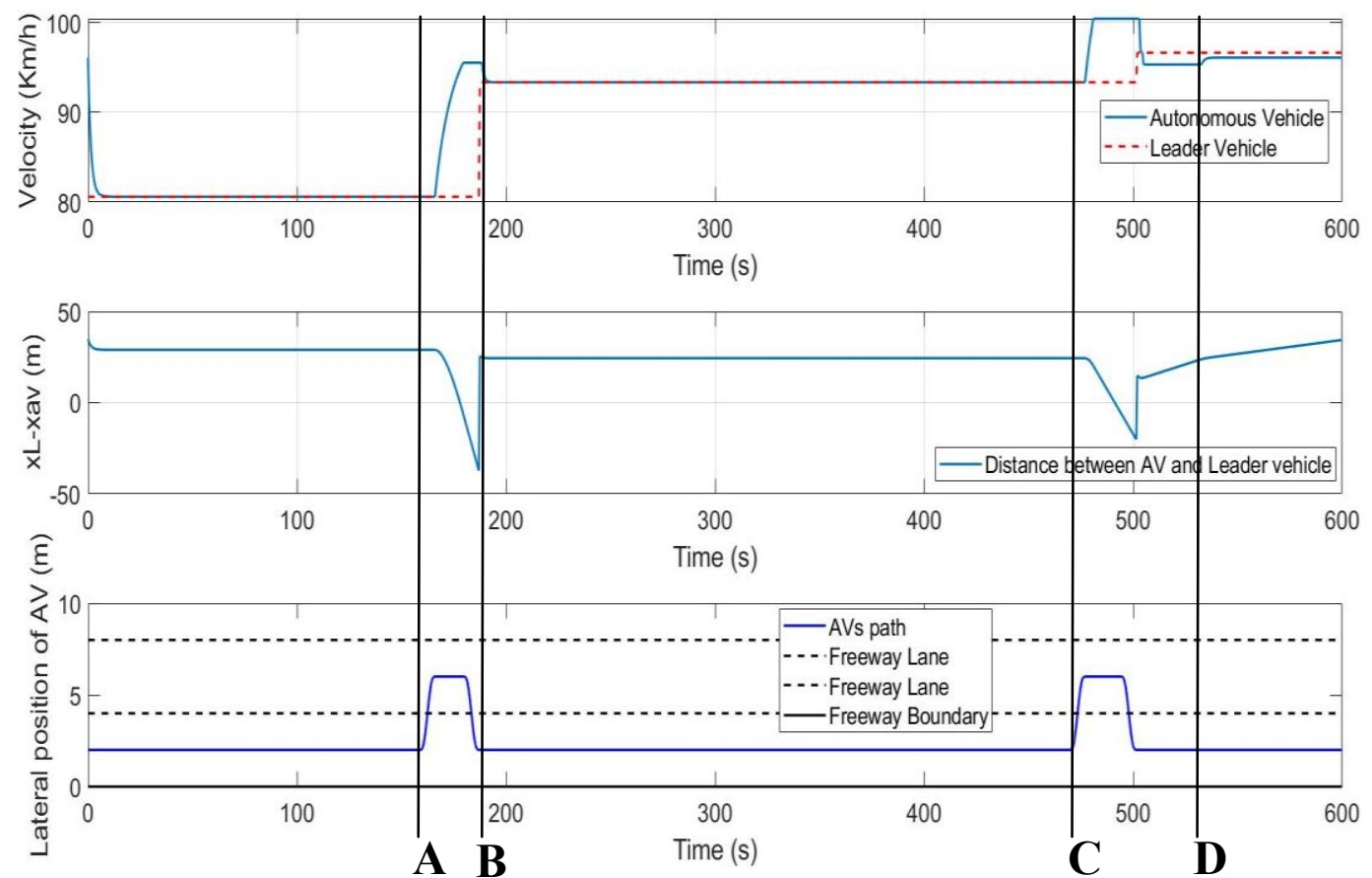

Figure 3. Above curve) velocity of AV and leader vehicle. Middle curve) relative distance between AV and leader vehicle. Bellow) longitudinal and lateral position of $\mathrm{AV}$

Table 1. First simulation results at $600 \mathrm{~s}(10 \mathrm{~min})$

\begin{tabular}{|c|c|}
\hline Number of lanes & 3 \\
\hline Default lane & Lane 1 (right lane) \\
\hline Desired speed $(\mathrm{Km} / \mathrm{h})$ & 96.09 \\
\hline Average speed $(\mathrm{Km} / \mathrm{h})$ & 90.51 \\
\hline Maximum acceleration $\left(\mathrm{m} / \mathrm{s}^{2}\right)$ & 0.63 \\
\hline Minimum acceleration $\left(\mathrm{m} / \mathrm{s}^{2}\right)$ & -2.6 \\
\hline Distance traveled $(\mathrm{Km})$ & 15.08 \\
\hline Speed adaptation & 2 \\
\hline Distance adaptation & 1 \\
\hline Free flow & 1 \\
\hline Number of overtaking & 2 \\
\hline
\end{tabular}

\subsection{Second simulation}

Second simulation is similar to the first simulation except that because of higher speed range of $\mathrm{AV}$, default lane is (lane 2) for moving. When program runs, according to freeway lane 2 speed limits, desired velocity $(116.3 \mathrm{Km} / \mathrm{h})$ has selected by program randomly. AV starts moving with this desired 
velocity in lane 2. At this time $(t=0 \mathrm{~s})$, two vehicles in the left lane with a randomly selected velocity and distance from AV is generated, and a LV with initial velocity $102.5 \mathrm{Km} / \mathrm{h}$ and initial distance 36.98 $\mathrm{m}$ in front of AV $\left(X_{f}\right)$ is generated. AV's velocity is higher than LV's velocity and $X_{f}$ is higher than $h_{m}$ (in second simulation $h_{m}=26.3 \mathrm{~m}$ ), AV checks distance between two vehicles $(D)$ in left lane for overtaking, and there is no safe distance $\left(D_{\text {safe }}\right)$ between two vehicles in left lane, so AV decelerates until its velocity reaches to $102.5 \mathrm{Km} / \mathrm{h}$ and $X_{f}$ reached to $32.48 \mathrm{~m}$ (speed adaptation is done). The AV keep moving with this velocity and distance $X_{f}$ from LV until overtaking conditions are provided. In section A, $(t=25.5 \mathrm{~s})$ safe distance $\left(D_{\text {safe }}\right)$ between two vehicles in left lane (lane 3$)$ is provided. Therefore, AV changes its lane and overtakes. During overtaking, AV's velocity reaches to $114.8 \mathrm{Km} / \mathrm{h}$. In section B, AV returns to previous lane (lane 2) and new vehicle with velocity $103.2 \mathrm{Km} / \mathrm{h}$ and distance $38.68 \mathrm{~m}$ in front of AV generates $(t=59 \mathrm{~s})$. AV's velocity is higher than LV's velocity and $X_{f}$ is higher than $h_{m}$, so $\mathrm{AV}$ decelerates and its velocity reaches to 103.2 until section $\mathrm{C}$ (speed adaptation is done). At this section overtaking conditions are provided and AV overtakes and its velocity reaches to $114.8 \mathrm{Km} / \mathrm{h}$. In section $\mathrm{D}$, AV returns to previous lane (lane 2) and new vehicle with velocity $113.1 \mathrm{Km} / \mathrm{h}$ and distance $14.09 \mathrm{~m}$ in the front of AV generates $(t=120.5 \mathrm{~s})$. AV decelerates and its velocity reaches to $113.0 \mathrm{Km} / \mathrm{h}$ $(0.1 \mathrm{Km} / \mathrm{h}$ is lower than LV's velocity), so keep moving with this velocity until distance between AV and $\mathrm{LV}$ reaches to $25.87 \mathrm{~m}$ (distance adaptation) and at $t=600 \mathrm{~s}$ program stops (Figure 4), result of this simulation is presented in Table 2 .

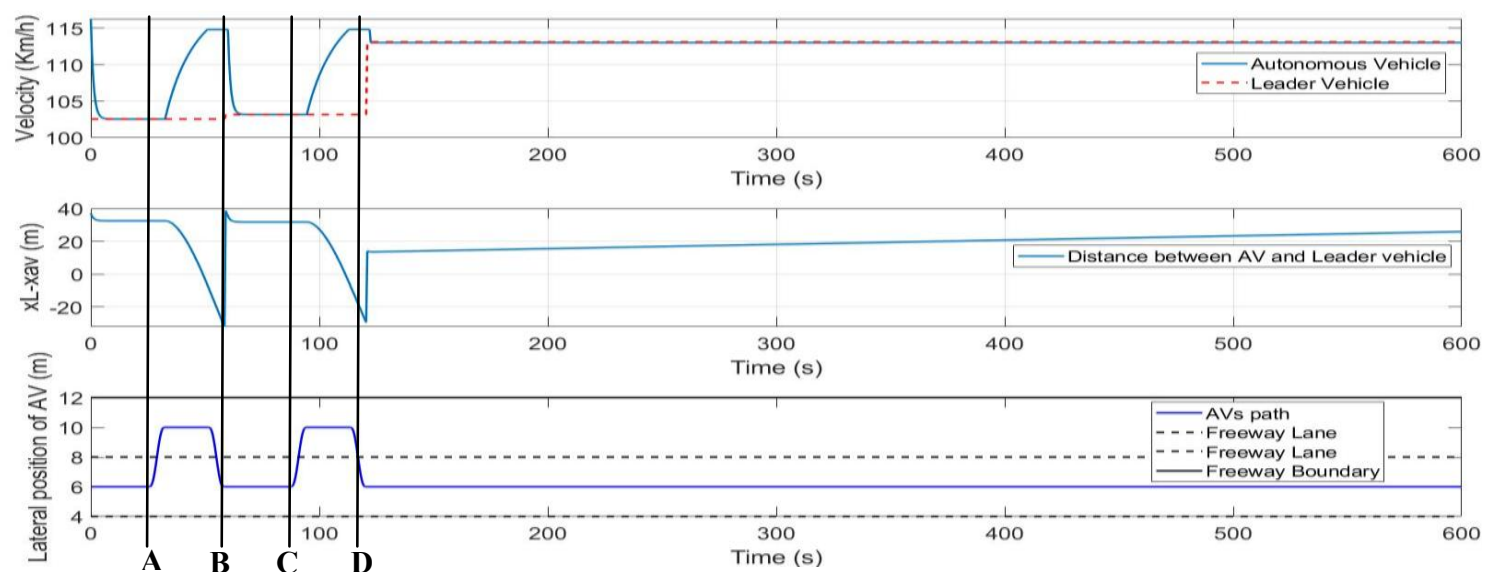

Figure 4. Above curve) velocity of AV and leader vehicle. Middle curve) relative distance between AV and leader vehicle. Bellow) longitudinal and lateral position of AV

Table 2. Second simulation results at $600 \mathrm{~s}(10 \mathrm{~min})$

\begin{tabular}{|c|c|}
\hline Number of lanes & 3 \\
\hline Default lane & Lane 2 (right lane) \\
\hline Desired speed $(\mathrm{Km} / \mathrm{h})$ & 116.3 \\
\hline Average speed $(\mathrm{Km} / \mathrm{h})$ & 111.8 \\
\hline Maximum acceleration $\left(\mathrm{m} / \mathrm{s}^{2}\right)$ & 0.36 \\
\hline Minimum acceleration $\left(\mathrm{m} / \mathrm{s}^{2}\right)$ & -2.67 \\
\hline Distance traveled $(\mathrm{Km})$ & 18.64 \\
\hline Speed adaptation & 2 \\
\hline Distance adaptation & 1 \\
\hline Free flow & 0 \\
\hline Number of overtaking & 2 \\
\hline
\end{tabular}

\section{Conclusion}

In this research trajectory planning of autonomous vehicle in a freeway is studied. Unlike most other researches that only analysed one type of driving modes like lane change curve or car following, this work analysed three types of driving modes free flow, car following, overtaking in a long-term driving. Formulations of all driving modes is presented. Decision making algorithm for performing all of the driving modes is presented. The 5th degree polynomial for lane change curvature is introduced and lateral acceleration of autonomous vehicle for passenger's comfort is considered. Two simulations are 
performed based on the decision-making algorithm to plan a desired trajectory considering peripheral vehicles relative velocities and distances with respect to AV. The Results of the simulations state that algorithm works correctly and finds appropriate trajectory for various road conditions.

\section{References}

1. Alotibi, F. Abdelhakim, M. (2020). Anomaly Detection for Cooperative Adaptive Cruise Control in Autonomous Vehicles Using Statistical Learning and Kinematic Model, IEEE Transactions on Intelligence Transportation Systems, 1-11, DOI : 10.1109/TITS.2020.2983392.

2. Bilik, I. Longman, O. Villeval, S. Tabrikian, J. (2019). The rise of radar for autonomous vehicles: signal processing solutions and future research directions, IEEE signal processing magazine, 36(5), 20-31.

3. Britting, K. R. (1999). Inertial Navigation Systems Analysis, Wiley, New York.

4. Dias, J. E. A. Pereira, G. A. S. and Palhares, R. M. (2015) Longitudinal Model Identification and Velocity Control of an Autonomous Car, IEEE Transactions on Intelligent Transportation Systems, 16(2), 776-786, DOI: 10.1109/TITS.2014.2341491.

5. Chen, B.-C. Bue, C.-C. (2014) Electronic Stability Control for Electric Vehicle with Four In-Wheel Motors, International Journal of Automotive Technology, 15(4), 573-580, DOI: 10.1007/s12239-014-0060-4.

6. Dixit S., Fallah S., Montanaro U., Dianati M., Stevens A., Mccullough F., and Mouzakitis A., (2018) Trajectory planning and tracking for autonomous overtaking: State-of-the-art and future prospects, Annual Rev. Control., 45, 76-86.

7. Ghariblu, H. (2020) Decision Making for Motion Planning of Autonomous Vehicles in the Freeway Driving, International Journal of Intelligent Transportation, Under review.

8. Kesting A., Treiber M., Schönhof M., Helbing D. Extending adaptive cruise control to adaptive driving strategies, Transportation Research Record, Vol. 2000 (1), pp. 16-24, 2007.

9. Houenou A., Bonnifait P., Cherfaoui V., and Wen Y. (2013). Vehicle Trajectory Prediction based on Motion Model and Maneuver Recognition. IEEE/RSJ International Conference on Intelligent Robots and Systems, Nov., 2013, Tokyo, Japan, 4363-4369.

10. Kanaris A., Kosmatopoulos E. B., and Loannou P. A., (2001). Strategies and spacing requirements for lane changing and merging in automated highway system", IEEE Trans. on Vehicular Technol., vol. 50(61), 586-1581.

11. Maksymova, I. Steger, C. Druml, N. (2018) Review of LiDAR Sensor Data Acquisition and Compression for Automotive Applications. Eurosensors 2018 Conferences, 2(13), 852, Austria.

12. Mammeri, A. Lu, G. Boukerche, A. (2015). Design of Lane Keeping Assist System for Autonomous Vehicle, 2015 7th International Conference on New Technologies, Mobility and Security (NTMS), 1-5, DOI : 10.1109/NTMS.2015.7266483.

13. Mampearachchi, W. K., Masakorala S. R. (2018). Analytical Model for Passing Sight Distance Design Criteria of Two-Lane Roads in Sri Lanka, Transport and Telecommunication Journal, 19(1), 10-20.

14. Milanés, V., Naranjo, J., González, C., Alonso, J., \& De Pedro, T. (2008). Autonomous vehicle based in cooperative GPS and inertial systems. Robotica, 26(5), 627-633. doi:10.1017/S0263574708004232.

15. Nelson, W. (1989) Continuous-curvature paths for autonomous vehicles, Proceedings of the International Conference on Robotics and Automation, Scottsdale, AZ, 3, 1260-1264 DOI: 10.1109/ROBOT.1989.100153.

16. Patole, S. M. Torlak, M. Wang, D. and Ali, M. (2017) Automotive radars: A review of signal processing techniques. IEEE Signal Processing Magazine, 34(2), 22-35, DOI: 10.1109/MSP.2016.2628914.

17. Sherif, M. Abuelenin, Adel Y. Abul-Magd, (2015) Effect of minimum headway distance on connectivity of VANETs, International Journal of Electronics and Communications, 69(5), 867-871, DOI: 10.1016/j.aeue.2015.01.011.

18. Shim, T., Adireddy, G., and Yuan, H. (2012) Autonomous vehicle collision avoidance system using path planning and model-predictive-control-based active front steering and wheel torque control, $P I$ Mech Eng D-J Aut., 226(6), 767-778.

19. Shojaei, S. Rahmani, H. A, Azadi, S., Saeedi MA. (2020) A novel decision-making unit for automated maneuver of articulated vehicles in real traffic situations. Proceedings of the Institution of Mechanical Engineers, Part D: Journal of Automobile Engineering, 234(1), 152-171. DOI: 10.1177/0954407019838380.

20. Skačkauskas, P., Sokolovskij, E. (2019) Analysis of the Hybrid Global Path Planning Algorithm for Different Environments, Transport and Telecommunication Journal, 20(1), 1-13. 
21. Sukkarieh, S. Nebot, E.M. Durrant-Whyte, H.F. (1999) A high integrity IMU/GPS navigation loop for autonomous land vehicle applications, IEEE Transactions on Robotics and Automation, 15(3), 572-578, DOI : 10.1109/70.768189.

22. Tarel, J. Hautiere, N. Caraffa, L. Cord, A. Halmaoui, H. and Gruyer, D. (2012) Vision Enhancement in Homogeneous and Heterogeneous Fog, IEEE Intelligent Transportation Systems Magazine, 4(2), 6-20, DOI: 10.1109/MITS.2012.2189969.

23. Wang, L. Zhang, Y.Wang, J. (2017) Map-based localization method for autonomous vehicles using 3D-lidar, IFAC PapersOnLine, 50(1), 276-281.

24. Yang, Q., Koutsopoulos, H. N. (1996) A microscopic traffic simulator for evaluation of dynamic traffic management systems, Transportation Research, Part C: Emerging Technologies, 4(3), 113-129.

25. Zaarane, A. Slimani, I. Al Okaishi, W. Atouf, I. Hamdoun, A. (2020) Distance measurement system for autonomous vehicles using stereo camera, Array. 5:100016, DOI: 10.1016/j.array.2020.100016.

26. Zhang, S., Deng, W., Zhao, Q., Sun, H., Litkouhi, B. (2013) Dynamic trajectory planning for vehicle autonomous driving. 2013 IEEE International Conference on Systems, Man, and Cybernetics, Oct., 2013, Manchester, UK, 4161-4166. 